\section{Cirugía coronaria: resultados inmediatos y alejados de la cirugía de revascularización miocárdica en enfermedad coronaria}

\author{
ROBERTO GONZÁLEZ L., ${ }^{1,2}$, RODRIGO REYES M. ${ }^{1,2}$, \\ ALECK STOCKINS L. ${ }^{1,2}$, ENRIQUE SEGUEL S..$^{1,2}$, \\ ANDRÉS JADUE T. ${ }^{1}$, EMILIO ALARCÓN C. ${ }^{1,2}$
}

\section{Early and late results of coronary artery bypass grafting in coronary artery disease in Concepcion, Chile}

Background: Coronary artery bypass grafting $(C A B G)$ is the best treatment for a large group of patients with coronary artery disease. Aim: To describe early and late results of patients treated with CABG at our Center. Patients and Methods: Revision of data bases, surgical protocols and clinical registers of patients operated between January 2006 and December 2008. Results: Of 1.003 cardiac surgeries performed during the period, 658 corresponded to isolated CABG (78\% in men). The median age of patients was 62 years. Left common coronary artery lesions was found in 135 cases (20\%), 555 patients (84\%) had hypertension and 231 (35\%) were diabetics. Four-hundred thirty (65\%) had stable angina and 211 (32\%) had a recent myocardial infarction. Twenty-two had left ventricular ejection fraction $<30 \%$. In 248 (38\%), a low Additive EuroSCORE risk was found. Emergency surgery was required in 36 (6\%) patients. Use of extracorporeal circulation was required in 466 patients (71\%). Within 30 days of surgery, complications were recorded in 105 patients (16\%) (15 (2\%) stroke, 14 (2\%) myocardial infarction, 7 (1\%) mediastinitis, and in $14(2 \%)$ a reoperation for bleeding). Thirteen patients died (2\%). Among the 303 patients with stable angina and preserved left ventricular function, two died (0,7\%). Survival at 1, 3 and 5 years was 97,4\%, 93,8\% y 90,9\% respectively. Major adverse cardiac and cerebrovascular events at five years occurred in 20\%, stroke in 4\%, myocardial infarction in 3\% and reintervention in $2 \%$. Seven percent of patients had recurrence of angina. Conclusions: We describe a heterogeneous series of patients. The immediate and late results are comparable with international communications.

(Rev Med Chile 2018; 146: 1395-1404)

Key words: Cardiac Surgical Procedures; Cardiopulmonary Bypass; Coronary Artery Bypass; Coronary Artery Disease.
${ }^{1}$ Centro Cardiovascular, Hospital Clínico Regional de Concepción: "Dr. Guillermo Grant Benavente". Concepción, Chile.

2Departamento de Cirugía, Facultad de Medicina, Universidad de Concepción, Concepción, Chile.

Trabajo no recibió apoyo financiero.

Los autores declaran no tener conflictos de interés.

Recibido el 23 de junio de 2018, aceptado el 11 de octubre de 2018.

Correspondencia a: Roberto González Lagos Departamento de Cirugía, Facultad de Medicina, Universidad de Concepción, Janequeo esquina Chacabuco S/N, Concepción, Chile. rgonzalezlagos@udec.cl
L a enfermedad coronaria (EC) corresponde a la primera causa de muerte por enfermedades cardiovasculares a nivel mundial y una importante causa de discapacidad ${ }^{1}$. En países desarrollados y en vías de desarrollo la EC es res- ponsable de un tercio de las muertes en personas de más de 35 años $^{2,3}$.

En los últimos 30 años se han realizado avances en el tratamiento médico y en técnicas de revascularización miocárdica quirúrgica o percutánea 
que han permitido disminuir la morbimortalidad asociada a la EC.

Desde su introducción masiva en $1968^{4}$, la cirugía coronaria (CC) se convirtió en el gold standard en el tratamiento de muchos pacientes con EC. Además, se trata del procedimiento quirúrgico que ha sido más extensa y meticulosamente estudiado en la literatura médica ${ }^{5,6}$ y ha demostrado beneficios en mejorar los síntomas de la EC, prevenir eventos clínicos y mejorar la supervivencia a largo plazo $^{7}$. Actualmente, es el tratamiento recomendado para un amplio espectro de pacientes con $\mathrm{EC}^{8,9}$.

El objetivo de este trabajo es presentar nuestra experiencia; mostramos los resultados inmediatos y alejados en los pacientes tratados con CC en nuestro hospital.

\section{Pacientes y Métodos}

Se incluyeron las CC aisladas realizadas entre enero de 2006 y diciembre de 2008 en Hospital Clínico Regional "Dr. Guillermo Grant Benavente" de Concepción-Chile. Se excluyeron CC con procedimientos adicionales.

Se revisó base de datos, fichas y protocolos. Para seguimiento clínico se revisaron registros hospitalarios y ficha clínica. Además, se realizó seguimiento clínico y/o telefónico buscando eventos cardiovasculares significativos. Se realizó seguimiento de supervivencia según datos disponibles en registro civil e identificación. Esta comunicación fue aprobada por el comité de ética de nuestra institución.

Como resultados inmediatos se consideró: mortalidad operatoria, infarto agudo al miocardio (IAM), accidente vascular encefálico (AVE), reoperaciones, transfusiones, insuficiencia renal aguda con hemodiálisis, morbilidad de herida operatoria de esternotomía y/o safenectomía (infecciones, seroma y dehiscencia), mediastinitis, estadía en cuidados intensivos (UCI) y estadía hospitalaria.

Para resultados alejados a 5 años se consideró supervivencia y Mayor-Adverse-Cardiac-and-Cerebrovascular-Events (MACCE) compuesto por: Muerte, IAM, AVE y reintervención coronaria (quirúrgica o percutánea).

Se utilizó Microsoft Excel 15.4 y software SPSS 24. Las variables categóricas se compararon utilizando $\chi^{2}$. Para comparación de supervivencia y eventos clínicos se utilizó Kaplan-Meier con log rank. Se consideró significativo $\mathrm{p}<0,05$.

\section{Resultados}

Se realizaron 1.003 cirugías cardiacas, 658 $(65,6 \%)$ fueron CC aisladas. Se utilizó circulación extracorpórea (CEC) en 466 (70,8\%), entre ellos, en 11 pacientes se realizó con CEC a corazón batiente. Se operaron sin CEC 192 (29,2\%) y entre ellos $6(3,1 \%)$ requirieron conversión.

La edad promedio fue $62,0 \pm 8,7$ años y de sexo masculino 516 (78,4\%).

Presentaron lesión de tronco coronario izquierdo $135(20,5 \%)$ y de tres vasos $476(72,3 \%)$, 460 pacientes presentaron una fracción de eyección de ventrículo izquierdo (FEVI) $>50 \%$.

Se evaluó riesgo utilizando EuroSCORE aditivo. La mediana del EuroSCORE fue 3 y presentaron riesgo bajo (EuroSCORE $\leq 2) 248(37,7 \%)$.

Las características clínicas y anatómicas de los pacientes se resumen en la Tabla 1.

\section{Resultados inmediatos (30 días)}

Se registraron resultados inmediatos en todos los pacientes (Tabla 2).

La mortalidad operatoria global fue $1,98 \%$. En el subgrupo de pacientes con angina estable y con FEVI $>50 \%(n=303)$ la mortalidad operatoria fue $0,66 \%$.

En los pacientes de bajo riesgo (EuroSCORE $\leq 2)$ la mortalidad fue $0,8 \%$. Según función ventricular izquierda, presentaron mortalidad el 1,08\% de los pacientes con FEVI > 50\%.

Presentaron IAM postoperatorio $14(2,1 \%)$ pacientes y AVE $15(2,3 \%)$. Existió morbilidad de herida en $20(3,0 \%)$ y mediastinitis en $7(1,1 \%)$. Cursaron con falla renal aguda con requerimiento de hemodiálisis $7(1,1 \%)$. Se reoperaron por hemorragia $14(2,1 \%)$. Requirieron transfusiones $143(21,7 \%)$. El número promedio de unidades de hemoderivados por paciente transfundido fue 5,4 Unidades/paciente.

La mediana en UCI fue 2 días y la mediana de estadía hospitalaria fue 6 días.

\section{Resultados alejados}

Se siguió supervivencia en todos los pacientes a través del registro civil e identificación, se excluyeron a pacientes con mortalidad operatoria (Ta- 
Tabla 1. Características clínicas y anatómicas de pacientes tratados con cirugía coronaria aislada

\begin{tabular}{|c|c|c|}
\hline \multicolumn{2}{|c|}{ Características clínicas } & $n=658$ \\
\hline \multicolumn{2}{|l|}{ Edad promedio (años) } & $62,0 \pm 8,7$ \\
\hline \multicolumn{2}{|c|}{ Estado nutricional eutrófico } & $165(25,1 \%)$ \\
\hline \multicolumn{2}{|l|}{ Sexo masculino } & $516(78,4 \%)$ \\
\hline \multirow[t]{3}{*}{ FEVI } & $>50 \%$ & $460(69,9 \%)$ \\
\hline & 30 a $50 \%$ & $176(26,7 \%)$ \\
\hline & $<30 \%$ & $22 \quad(3,3 \%)$ \\
\hline \multicolumn{2}{|l|}{ Angina } & $643(97,7 \%)$ \\
\hline \multicolumn{2}{|l|}{ Angina inestable } & $213(32,4 \%)$ \\
\hline \multicolumn{2}{|l|}{ Hipertensión arterial } & $555(84,3 \%)$ \\
\hline \multicolumn{2}{|l|}{ Dislipidemia } & $285(43,3 \%)$ \\
\hline \multicolumn{2}{|l|}{ Tabaquismo } & $259(39,4 \%)$ \\
\hline \multicolumn{2}{|l|}{ Diabetes mellitus } & $231(35,1 \%)$ \\
\hline \multicolumn{2}{|l|}{ EAOC } & $53(8,1 \%)$ \\
\hline \multicolumn{2}{|l|}{ EPOC } & $41 \quad(6,2 \%)$ \\
\hline \multicolumn{2}{|c|}{ Enfermedad renal crónica } & $20 \quad(3,0 \%)$ \\
\hline \multicolumn{2}{|l|}{ IAM (< 90 días) } & $211(32,1 \%)$ \\
\hline \multicolumn{2}{|l|}{ AVE previo } & $28(4,3 \%)$ \\
\hline \multicolumn{2}{|l|}{ Angioplastía previa } & $93(14,1 \%)$ \\
\hline \multicolumn{2}{|l|}{ Cirugía de urgencia } & $36(5,5 \%)$ \\
\hline $\begin{array}{l}\text { Riesgo quirúrgico } \\
\text { (EuroSCORE } \\
\text { aditivo) }\end{array}$ & $\begin{array}{l}\text { Promedio } \\
\text { Bajo }(\leq 2) \\
\text { Moderado (3 a } 5) \\
\text { Alto }(\geq 6)\end{array}$ & $\begin{array}{c}3,56 \pm 2,5 \\
248(37,7 \%) \\
273(41,5 \%) \\
137(20,8 \%)\end{array}$ \\
\hline \multicolumn{3}{|c|}{ Características anatómicas } \\
\hline \multicolumn{2}{|c|}{ Lesión tronco común izquierdo } & $135(20,5 \%)$ \\
\hline \multirow[t]{4}{*}{$\mathrm{n}$ de vasos } & Promedio & $2,6 \pm 0,7$ \\
\hline & 1 vaso & $53(8,1 \%)$ \\
\hline & 2 vasos & $129(19,6 \%)$ \\
\hline & 3 vasos & $476(72,3 \%)$ \\
\hline \multicolumn{2}{|l|}{ Pared anterior } & $654(99,4 \%)$ \\
\hline \multicolumn{2}{|l|}{ Pared lateral } & $574(87,2 \%)$ \\
\hline \multicolumn{2}{|l|}{ Pared inferior } & $486(73,9 \%)$ \\
\hline \multicolumn{2}{|l|}{ n Puentes (promedio) } & $3,2 \pm 1,0$ \\
\hline \multicolumn{3}{|l|}{ Conductos } \\
\hline \multicolumn{2}{|c|}{ Arteria mamaria interna izquierda } & $642(97,6 \%)$ \\
\hline \multicolumn{2}{|l|}{ Dos o más arterias } & $27 \quad(4,1 \%)$ \\
\hline \multicolumn{2}{|l|}{ Vena safena interna } & $606(92,1 \%)$ \\
\hline
\end{tabular}

FEVI: Fracción de eyección del ventrículo izquierdo; EPOC: Enfermedad pulmonar obstructiva crónica; EAOC: Enfermedad arterial obstructiva crónica; IAM: Infarto agudo al miocardio; AVE: Accidente vascular encefálico.
Tabla 2. Resultados a 30 días de pacientes tratados con cirugía coronaria aislada

\begin{tabular}{|c|c|c|}
\hline & n (\%) & $\mathbf{p}$ \\
\hline Mortalidad global $(n=658)$ & $13(1,98 \%)$ & \\
\hline $\begin{array}{l}\text { Mortalidad con FEVI normal y } \\
\text { angina estable }(n=303)\end{array}$ & $2(0,66 \%)$ & \\
\hline \multicolumn{3}{|c|}{ Mortalidad según riesgo quirúrgico } \\
\hline EuroSCORE $\leq 2(n=248)$ & $2(0,80 \%)$ & \\
\hline EuroSCORE 3 a $5(n=273)$ & $4(1,50 \%)$ & 0,01 \\
\hline EuroSCORE $\geq 6(n=137)$ & $7(5,10 \%)$ & \\
\hline \multicolumn{3}{|l|}{ Mortalidad según FEVI } \\
\hline $\mathrm{FEVI}>50 \%(n=460)$ & $5(1,08 \%)$ & \\
\hline FEVI 30 a $50 \%(n=176)$ & $7(3,97 \%)$ & 0,04 \\
\hline FEVI $<30 \%(n=22)$ & $1(4,55 \%)$ & \\
\hline \multicolumn{3}{|l|}{ Morbilidad } \\
\hline Morbilidad de herida operatoria & $20(3,0 \%)$ & \\
\hline AVE postoperatorio & $15(2,3 \%)$ & \\
\hline IAM postoperatorio & $14(2,1 \%)$ & \\
\hline Reoperación por hemorragia & $14(2,1 \%)$ & \\
\hline Mediastinitis & $7(1,1 \%)$ & \\
\hline Insuficiencia renal (hemodiálisis) & $7(1,1 \%)$ & \\
\hline Necesidad de transfusión & $143(21,7 \%)$ & \\
\hline \multicolumn{3}{|l|}{ Estadía UCI (días) } \\
\hline Mediana & 2 & \\
\hline Rango & $1-29$ & \\
\hline \multicolumn{3}{|l|}{ Estadía hospitalaria (días) } \\
\hline Mediana & 6 & \\
\hline Rango & $3-71$ & \\
\hline
\end{tabular}

FEVI: Fracción de eyección del ventrículo izquierdo; AVE: accidente vascular encefálico; IAM: Infarto agudo al miocardio; UCl: Unidad de cuidados intensivos.

bla 3). Supervivencia global a 1, 3 y 5 años fue de 97,4\%, 93,8\% y 90,9\% respectivamente (Figura 1).

Se analizó supervivencia a 5 años según FEVI, encontrando diferencias entre los grupos $(\mathrm{p}<0,001)$ (Figura 2).

Se logró seguimiento clínico completo en $548(83,3 \%)$ pacientes. Entre estos, ocurrieron MACCE a 1, 3 y 5 años en 10,5\%, 15,1\% y 19,7\% respectivamente (Figura 3 ). Recurrencia de angina en seguimiento en $37(6,8 \%)$ pacientes.

Según función ventricular, encontramos diferencias entre los grupos $(p=0,001)$ (Figura 4). 


\section{Discusión}

A partir de los primeros casos en 1967 en la Cleveland Clinic $^{10}$ la CC experimentó un aumento explosivo, expandiéndose a nivel mundial y siendo considerada una de las mayores revoluciones

\section{Tabla 3. Resultados alejados de pacientes} tratados con cirugía coronaria aislada

\begin{tabular}{|c|c|c|}
\hline & & $\mathbf{p}$ \\
\hline \multicolumn{3}{|l|}{ Supervivencia global } \\
\hline 1 año & $97,4 \%$ & \\
\hline 3 años & $93,8 \%$ & \\
\hline 5 años & $90,9 \%$ & \\
\hline \multicolumn{3}{|c|}{ Supervivencia a 5 años según FEVI } \\
\hline $\mathrm{FEVI}>50 \%(\mathrm{n}=460)$ & $93,5 \%$ & \\
\hline FEVI 30 a $50 \%(n=176)$ & $86,9 \%$ & $<0,001$ \\
\hline $\mathrm{FEVI}<30 \%(\mathrm{n}=22)$ & $68,2 \%$ & \\
\hline \multicolumn{3}{|l|}{ Eventos clínicos a 5 años } \\
\hline MACCE & $19,7 \%$ & \\
\hline AVE & $3,5 \%$ & \\
\hline IAM & $2,7 \%$ & \\
\hline Reintervención & $2,0 \%$ & \\
\hline Recurrencia de angina & $6,8 \%$ & \\
\hline
\end{tabular}

FEVI: Fracción de eyección del ventrículo izquierdo; MACCE: Mayor-adverse-Cardiac-and-Cerebrovascular-events; AVE: Accidente vascular encefálico; IAM: Infarto agudo al miocardio. médicas del siglo XX. Entre otros hitos, fue uno de los primeros procedimientos en ser evaluado con estudios randomizados, demostrando beneficios respecto a supervivencia y alivio de angina al compararse con tratamiento médico en angina estable $^{11}$. La CC se ha convertido en el procedimiento quirúrgico más estudiado en la literatura médica. El gran volumen de información ha permitido conocer sus complicaciones y resultados e identificar factores asociados a morbimortalidad inmediata y alejada ${ }^{12}$.

La seguridad y eficacia de la CC ha sido confirmada en múltiples estudios randomizados y meta-análisis. Ha demostrado superioridad en determinados grupos de pacientes al ser comparada con el tratamiento médico y percutáneo. Las guías clínicas recientes de distintas sociedades respaldan a la CC como el tratamiento de elección para un amplio espectro de pacientes con $\mathrm{EC}^{13,14}$.

Al compararla con la revascularización percutánea, la CC ha demostrado superioridad en supervivencia y eventos clínicos en diversas series y estudios randomizados. Uno de los estudios ranzomizados más relevantes es el SYNTAX, donde participaron 85 centros en Estados Unidos y Europa y se randomizaron a 1.800 pacientes con enfermedad de tronco común o tres vasos a CC o intervención percutánea utilizando stent medicado. A 5 años la supervivencia en los pacientes quirúrgicos fue $88,6 \%$ y presentaron MACCE

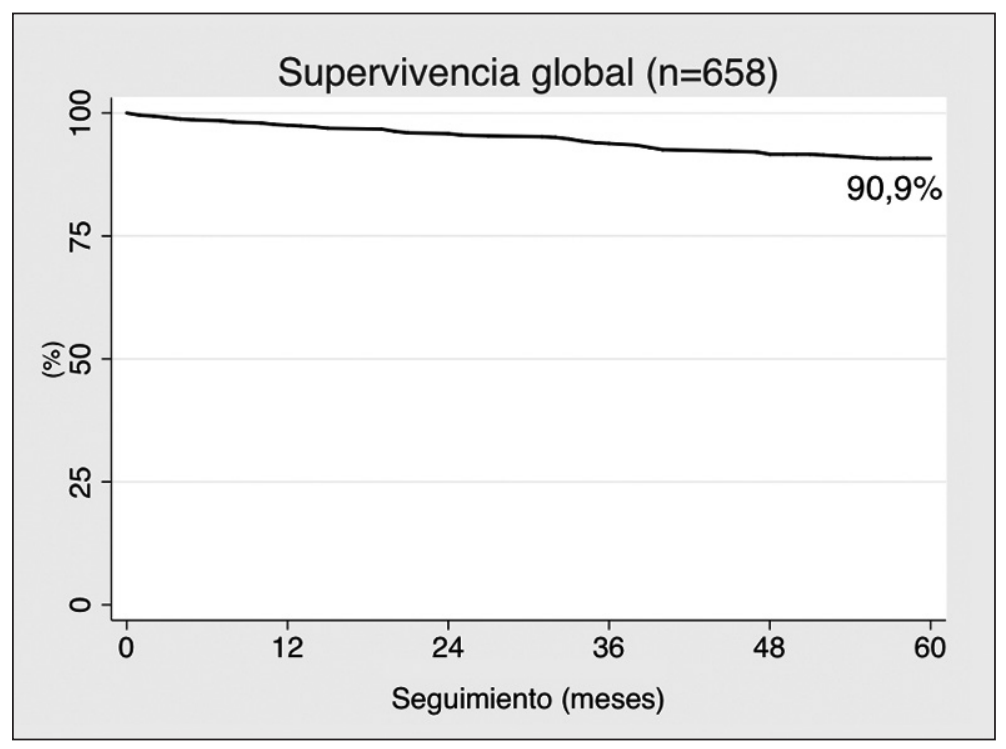

Figura 1. Supervivencia global de pacientes tratados con cirugía coronaria aislada. 


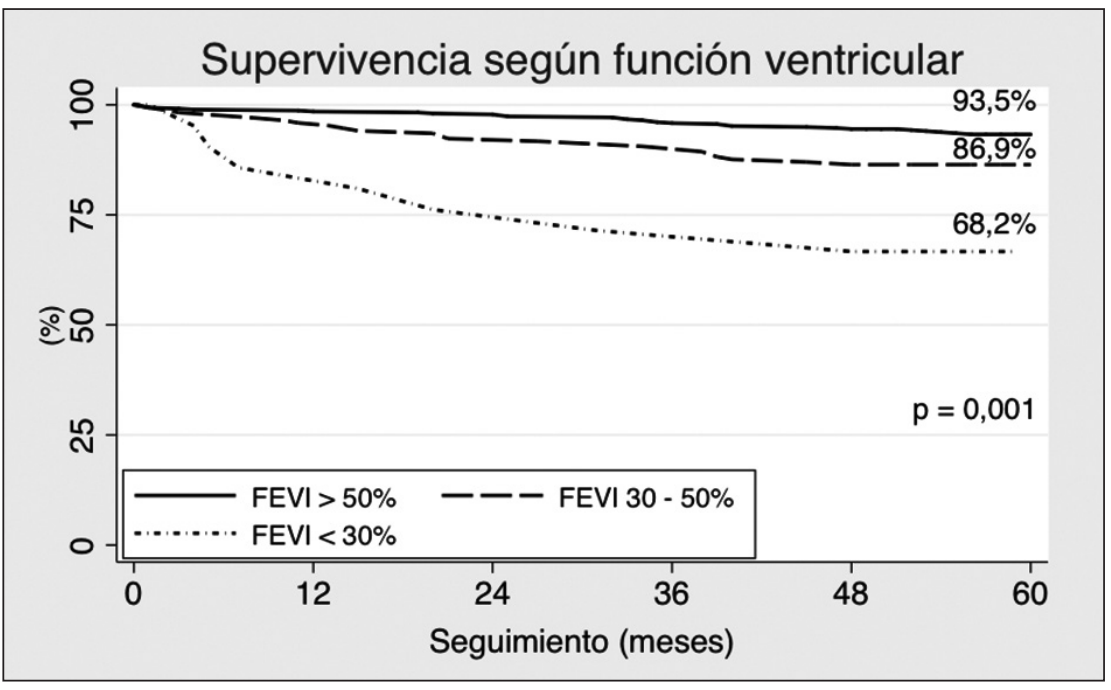

Figura 2. Supervivencia según función de ventrículo izquierdo de pacientes tratados con cirugía coronaria aislada $(\mathrm{FEVI}=$ Fracción de eyección del ventrículo izquierdo).

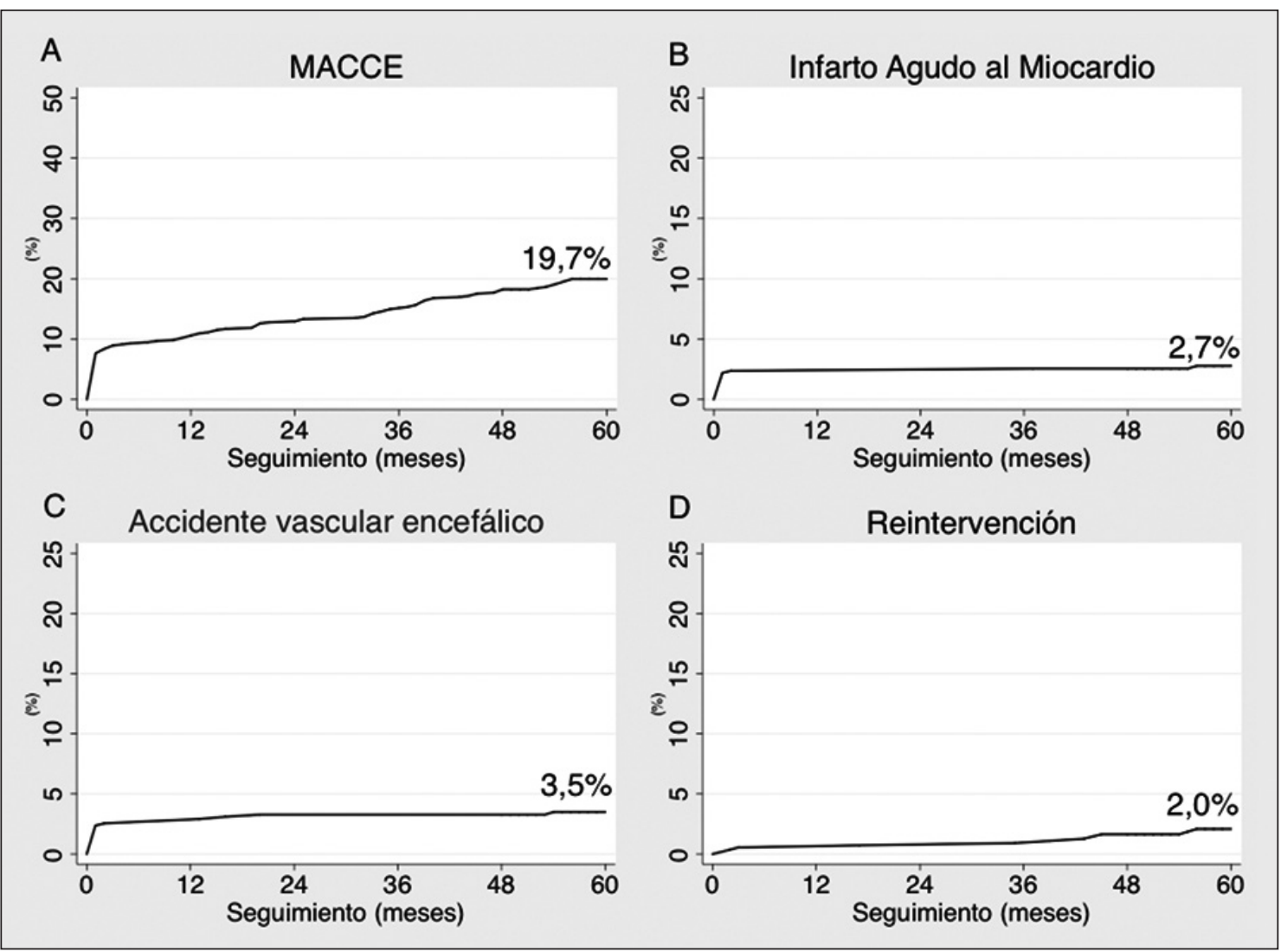

Figura 3. Eventos clínicos a 5 años: A) Mayor-adverse-Cardiac-and-Cerebrovascular-events (MACCE); B) Infarto agudo al miocardio; C) Accidente vascular encefálico; D) Reintervención. 


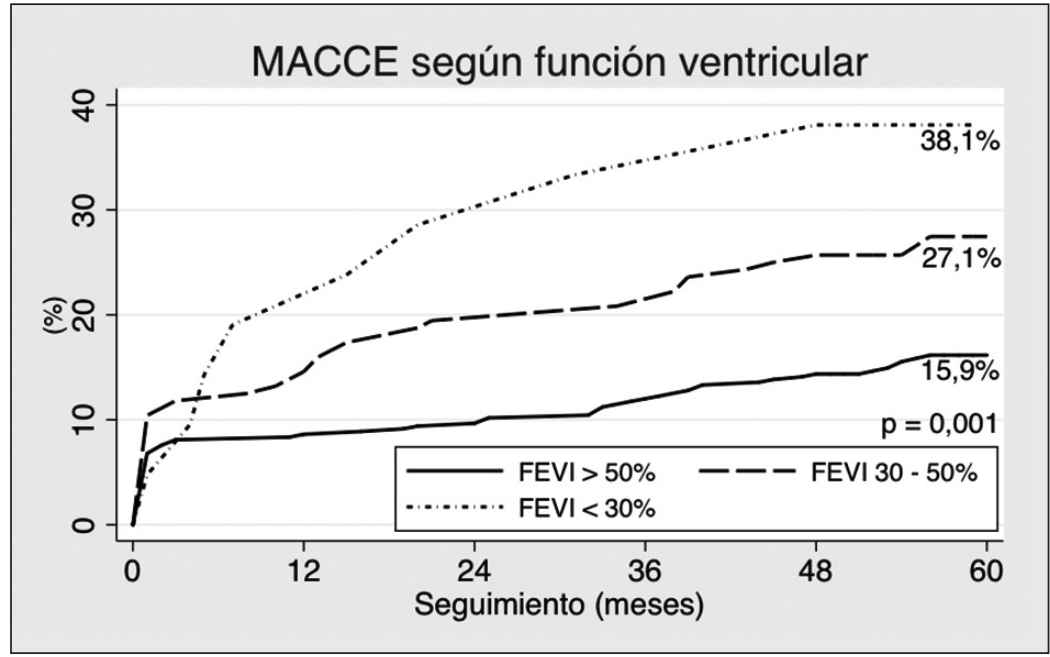

Figura 4. Mayor-adverse-Cardiac-and-Cerebrovascular-events (MACCE) según función de ventrículo izquierdo (FEVI: Fracción de eyección de ventrículo izquierdo).
$26,9 \%$. Entre estos, IAM en 3,8\%, AVE en 2,4\% y reintervención $13,7 \%$. La CC mostró superioridad al tratamiento percutáneo en relación a supervivencia y eventos clínicos ${ }^{15}$.

En las últimas décadas se ha producido un cambio en el perfil de pacientes tratados con $\mathrm{CC}^{16}$. La edad promedio era de 50-55 años, predominantemente hombres y los pacientes diabéticos no superaban el 30\% ${ }^{17,18,19}$. La edad, comorbilidades y riesgo quirúrgico de los pacientes que requieren $\mathrm{CC}$ han aumentado en las últimas décadas. A pesar de esto, la mortalidad operatoria ha disminuido constantemente hasta 1 a $3 \%$ en series recientes. Distintos avances, entre ellos la protección miocárdica y la prevención secundaria de la EC han contribuido disminuir la morbimortalidad asociada a la CC y a mejorar los resultados alejados ${ }^{20}$.

En relación a los conductos utilizados, se ha demostrado una mejor permeabilidad de conductos arteriales por sobre venosos y distintos estudios han evaluado sus resultados alejados ${ }^{21,22}$. En 1986, Loop y cols demostraron superioridad del injerto de arteria mamaria interna izquierda (AMI) a arteria descendente (DA) anterior por sobre vena safena, respecto a supervivencia y eventos clínicos $^{23}$. Actualmente, la anastomosis de AMI a DA y puentes aorto-coronarios con vena safena a los demás objetivos es la estrategia de revascularización más frecuente ${ }^{24} \mathrm{y}$ en nuestra serie fue la estrategia utilizada en la gran mayoría. Durante el período estudiado, nuestro equipo realizaba safenectomía con técnica de disección abierta, la cual no está exenta de morbilidad asociada a la herida operatoria, sobre todo en obesos, diabéticos o portadores de enfermedad arterial periférica. A mediados de los 90' apareció la técnica de disección endoscópica ${ }^{25}$ que ha mostrado reducir la morbilidad (hematoma, seroma, infección, dolor y parestesias) asociada a la safenectomía y mejorar resultados estéticos ${ }^{26}$. Desde el 2015 nuestro equipo incorporó la safenectomía endoscópica, utilizándola actualmente en la mayoría de los casos.

El uso un segundo conducto arterial se ha asociado a una ventaja en supervivencia con respecto a los conductos venosos en pacientes selecciona$\operatorname{dos}^{27-29}$. Series actuales han reportado el uso de dos o más conductos arteriales en aproximadamente $10 \%$ en grandes centros ${ }^{30}$. En nuestra serie se utilizó más de un conducto arterial en 4,1\% de los pacientes. En los últimos años, nuestro equipo ha aumentado el uso de anastomosis arteriales y, según datos obtenidos de nuestro registro prospectivo, actualmente hemos aumentado el uso de conductos arteriales y realizamos 2 o más anastomosis arteriales en $14,9 \%$ de los pacientes, lo que podría contribuir a mejorar nuestros resultados a largo plazo.

Una de las variantes técnicas a la cirugía coronaria convencional es la cirugía sin CEC. En series internacionales actuales la cirugía sin CEC representa aproximadamente 15 a $20 \%$ de los pacientes operados ${ }^{31}$. En nuestro equipo se adoptó 
la técnica de la cirugía sin CEC a mediados de la década pasada y se aumentó progresivamente su uso en pacientes de mayor riesgo quirúrgico y con mayor número de vasos enfermos ${ }^{32,33}$. En nuestra serie se operaron sin CEC 29,2\% de los pacientes. Aún existe controversia sobre los beneficios, seguridad y resultados a largo plazo de la cirugía sin CEC. En la actualidad realizamos CC sin CEC en casos seleccionados a criterio del cirujano.

Se han diseñado múltiples escalas para estimar el riesgo de los tratados con CC. En el período estudiado, nuestro equipo utilizó el EuroSCORE, el cual ha sido validado como predictor de mortalidad operatoria ${ }^{34,35}$. Según este, los pacientes con menos de 3 puntos se clasifican como bajo riesgo, entre 3 a 5 puntos como riesgo intermedio y con más de 5 como alto riesgo. La mayoría de nuestros pacientes presentaron riesgo quirúrgico intermedio. Al comparar la mortalidad operatoria entre los distintos niveles de riesgo existieron diferencias significativas. Hoy en día, se encuentra disponible el EuroSCORE II $^{36} \mathrm{y}$ actualmente, nuestro equipo lo utiliza de rutina.

Dado que la disfunción ventricular puede deberse en parte a miocardio hibernado o aturdido, la CC tiene la posibilidad de mejorar la función ventricular en estos pacientes. Entre las opciones de tratamiento quirúrgico de estos pacientes está la CC, la remodelación ventricular y el trasplante cardiaco, el último limitado por la disponibilidad de donantes. Estudios clásicos han demostrado que el tratamiento médico exclusivo se asocia a malos resultados a largo plazo y que la CC tiene ventajas en supervivencia global y en reducción de eventos $\operatorname{clínicos}^{37-39}$. Entre la literatura reciente, en el estudio STITCH se randomizaron a 1.212 pacientes con FEVI $<35 \%$ a tratamiento médico exclusivo o tratamiento médico y $\mathrm{CC}^{40}$. Los pacientes del grupo quirúrgico mostraron ventajas respecto a supervivencia global, muerte por causa cardiaca y hospitalizaciones de causa cardiaca ${ }^{41}$. Sin embargo, la presencia de disfunción ventricular izquierda es un factor de riesgo importante para los resultados a corto y largo plazo de la $\mathrm{CC}^{42}$. Distintas publicaciones la identifican como un predictor independiente de mortalidad operatoria ${ }^{43,44} \mathrm{y}$, en nuestra serie se asoció significativamente a una mayor mortalidad. Al evaluar el impacto de la disfunción ventricular en la supervivencia a 5 años, también encontramos diferencias significativas.

A nivel nacional, la cirugía coronaria se realiza en distintos centros a lo largo del país. Sin embargo, los resultados a corto y largo plazo solo han sido reportados por algunos centros. El equipo del Hospital Clínico de la Pontificia Universidad Católica de Chile ha sido constante en comunicar sus resultados. Irarrázaval y cols, presentaron los resultados del seguimiento precoz y alejado de pacientes tratados con CC mostrando excelentes resultados inmediatos y alejados ${ }^{45}$. Morán y cols, presentaron una comparación de resultados clínicos y seguimiento alejado entre pacientes seleccionados en quienes se utilizó una o dos arterias mamarias internas, mostrando una ventaja del uso de doble arteria mamaria en cuando a sobrevida libre de infarto y de angina ${ }^{46}$.

Logramos seguimiento para supervivencia en todos los pacientes gracias a los datos disponibles en el registro civil e identificación. A 5 años la supervivencia de nuestra serie fue de 90,9\% lo que es comparable a resultados de grandes registros internacionales y estudios randomizados con seguimiento a largo plazo.

Para el seguimiento de eventos clínicos revisamos extensamente los registros hospitalarios disponibles y se intentó contacto telefónico en todos los pacientes logrando un seguimiento clínico completo, ya sea por entrevista médica o cuestionario telefónico en $83,3 \%$ de la serie. Sin embargo, es posible que exista subdiagnóstico de eventos clínicos comparado con los estudios randomizados y grandes cohortes, ya que el control clínico y el acceso a pruebas diagnósticas y reintervenciones no es igual en nuestro medio que en centros del primer mundo. Además, en nuestro hospital no se realizan de rutina controles angiográficos, sino que estos son motivados por eventos clínicos. Una de nuestras ventajas es que, durante el período estudiado, la gran mayoría de los pacientes operados provenían de la región del Bío Bío y su seguimiento se realizaba en nuestro hospital. Actualmente, nuestro equipo se ha transformado en un referente que recibe pacientes de distintas ciudades de Chile, estos pacientes continúan su seguimiento alejado en sus centros de origen, dificultando el seguimiento clínico en el largo plazo.

En los últimos años, al igual que lo reportado en series internacionales, se ha producido un cambio en las características de la CC en nuestro hospital. Según nuestro registro prospectivo, entre los años 2015 y 2017, nuestro equipo realizó en total 
1.512 cirugías cardiacas, de las cuales 761 (50,3\%) corresponden a CC aislada. Esto representa un aumento en el número absoluto de CC, si bien la proporción de CC aislada es menor, lo que se debe al aumento de cirugías combinadas y valvulares. Actualmente, ha aumentado significativamente la edad promedio de nuestros pacientes, el número de pacientes diabéticos, con enfermedad renal crónica, disfunción ventricular, portadores de lesión de tronco común izquierdo y operados en contexto de un IAM reciente. Esto se ve reflejado en que el riesgo quirúrgico de nuestros pacientes estimado con EuroSCORE actualmente es significativamente mayor $(3,6$ vs 4,$1 ; \mathrm{p}<0,05)$. Con respecto a la técnica quirúrgica, hemos disminuido el uso de cirugía sin CEC, lo que en parte de debe a la mayor complejidad, tanto de las características clínicas como de las lesiones coronarias. En relación a los conductos utilizados hemos aumentado el uso de un segundo conducto arterial. Dentro de los desafíos a mediano plazo está intentar realizar el seguimiento a 10 años de este grupo de pacientes, así como, evaluar los resultados actuales de la CC en nuestro hospital. El cambio que ha experimentado el perfil de paciente tratado con CC, hacia una mayor complejidad y gravedad, nos obliga a evaluar y comunicar nuestros resultados constantemente.

A modo de conclusión, nuestra serie incluye pacientes de distintos niveles de riesgos y presenta resultados inmediatos y alejados que son comparables a grandes series internacionales.

\section{Referencias}

1. Heart Disease and Stroke Statistics - 2018 Update A Report From the American Heart Association. Circulation 2018; 137: e67-e492.

2. Lozano R, Naghavi M, Foreman K, Lim S, Shibuya K, Aboyans V, et al. Global and regional mortality from 235 causes of death for 20 age groups in 1990 and 2010: A systematic analysis for the Global Burden of Disease Study 2010. Lancet 2012; 380: 2095-128.

3. Nichols M, Townsend N, Scarborough P, Rayner M. Cardiovascular disease in Europe 2014: epidemiological update. Eur Heart J 2014; 35: 2950-9.

4. Favaloro RG. Saphenous vein autograft replacement of severe segmental coronary artery occlusion: operative technique. Ann Thorac Surg 1968; 5: 334-9.

5. Jones D. CABG at 50 (or 107?) - The complex course of therapeutic innovation. N Engl J Med 376; 19: 1809-11.

6. Zalaquett R. 50 años de cirugía de bypass coronario. Meditar el pasado, enfrentar el presente y forjar el futuro. Rev Chil Cardiol 2017; 36: 162-9.

7. Yusuf S, Zucker D, Peduzzi P, Fisher LD, Takaro T, Kennedy JW, et al. Effect of coronary artery bypass surgery on survival: overview of 10 -years results from randomised trials by the coronary artery bypass graft surgery trialists collaboration. Lancet 1994; 344: 563-70.

8. Montalescot G, Sechtem U, Achenbach S, Andreotti F, Arden C, Budaj A, et al. ESC guidelines on the management of stable coronary artery disease: The Task Force on the management of stable coronary artery disease of the European Society of Cardiology. Eur Heart J 2013; 34: 2949-3003.

9. Jadue A, González R, Irarrázaval MJ. Cirugía de revascularización miocárdica versus angioplastía coronaria con stent en enfermedad de tres vasos y/o tronco común izquierdo en diabéticos: meta-análisis de estudios aleatorios. Rev Med Chile 2012; 140: 640-8.

10. Favaloro RG, Effler DB, Cheanvechai C, Quint RA, Sones FM Jr. Acute coronary in sufficiency (impending myocardial infarction and myocardial infarction): surgical treatment by the saphenous vein graft technique. Am J Cardiol 1971; 28: 598-607.

11. Takaro T, Hultgren HN, Lipton MJ, Detre KM, Participants in the VA Study Group: The VA Cooperative Randomized Study of Surgery for Coronary Arterial Occlusive Disease: I. Subgroup with significant left main lesions. Circulation 1976; 54 (suppl I): I-107-I-17 (The Veterans Administration Coronary Artery Bypass Surgery Cooperative Study Group: Eleven-year survival in the Veterans Administration randomized trial of coronary bypass surgery for stable angina. $\mathrm{N}$ Engl J Med 1984; 311: 1333-9.

12. Head S, Kieser T, Falk V, Huysmans H, Kappetein P. Coronary artery bypass grafting: part 1 - the evolution overt the first 50 years. Eur Heart J 2013; 34: 2862-72.

13. Kolh P, Windecker S, Alfonso F, Collet JP, Cremer J, Falk V, et al. 2014 ESC/EACTS guidelines on myocardial revascularization: the task force on myocardial revascularization of the European Society of Cardiology (ESC) and the European Association for Cardio-Thoracic Surgery (EACTS). Developed with the special contribution of the European Association of Percutaneous Cardiovascular Interventions (EAPCI). Eur J Cardiothorac Surg 2014; 46: 517-92.

14. Hillis LD, Smith PK, Anderson JL, Bittl JA, Bridges CR, Byrne JG, et al. 2011 ACCF/AHA guideline for coronary artery bypass graft surgery. A report of the American College of Cardiology Foundation/American Heart 
Association Task Force on Practice Guidelines. Developed in collaboration with the American Association for Thoracic Surgery, Society of Cardiovascular Anesthesiologists, and Society of Thoracic Surgeons. J Am Coll Cardiol 2011; 58: 123-210.

15. Mohr FW, Morice MC, Kappetein AP, Feldman TE, Stahle E, Colombo A. Coronary artery bypass graft surgery versus percutaneous coronary intervention in patients with three-vessel disease and left main coronary disease: 5-year follow-up of the randomised, clinical SYNTAX trial. Lancet 2013; 381: 629-38.

16. Head SJ, Howell NJ, Osnabrugge RL, Bridgewater B, Keogh BE, Kinsman R. The European Association for Cardio-Thoracic Surgery (EACTS) database: an introduction. Eur J Cardiothorac Surg 2013; 44: 175-80.

17. Cooley DA, Dawson JT, Hallman GL, Sandiford FM, Wukasch DC, García E. Aortocoronary saphenous vein bypass. Results in 1492 patients, with particular reference to patients with complicating features. Ann Thorac Surg 1973; 16: 380-90.

18. Yusuf S, Zucker D, Peduzzi P, Fisher LD, Takaro T, Kennedy JW. Effect of coronary artery bypass graft surgery on survival: overview of 10-year results from randomised trials by the Coronary Artery Bypass Graft Surgery Trialists Collaboration. Lancet 1994; 344: 563-70.

19. Verska JJ, Walker WJ. Aortocoronary bypass in the diabetic patient. Am J Cardiol 1975; 35: 774-7.

20. Favaloro RG. Critical analysis of coronary artery bypass graft surgery: a 30-year journey. J Am Coll Cardiol 1998; 31: 1B-63B.

21. Fitzgibbon GM, Kafka HP, Leach AJ, Keon WJ, Hooper GD, Burton JR. Coronary bypass graft fate and patient outcome: angiographic follow-up of 5,065 grafts related to survival and reoperation in 1,388 patients during 25 years. J Am Coll Cardiol 1996; 28: 616-26.

22. Campeau L, Enjalbert M, Lespérance J, Bourassa MG, Kwiterovich P, Wachilder S, et al. The relation of risk factors to the development of atherosclerosis in saphenous-vein bypass grafts and the progression of disease in the native circulation. A study 10 years after aortocoronary bypass surgery. N Engl J Med 1984; 311: 1329-32.

23. Loop FD, Lytle BW, Cosgrove DM, Stewart RW, Goormastic M, Williams GW, et al. Influence of the internal mammary artery graft on 10-year survival and other cardiac events. N Engl J Med 1986; 314: 1-6.

24. Goldman S, Zadina K, Moritz T, Ovitt T, Sethi G, Copeland JG. V. A. Cooperative Study Group. Long-term patency of saphenous vein and left internal mammary artery grafts after coronary artery bypass surgery: results from a Department of Veterans Affairs Cooperative Study. J Am Coll Cardiol 2004; 44: 2149-56.
25. Lumsden AB, Eaves FF III, Ofenloch JC, Jordan WD. Subcutaneous, video-assisted saphenous vein harvest: report of the first 30 cases. Cardiovasc Surg 1996; 4: 771-6.

26. Dacey LJ. Endoscopic vein-graft harvest is safe for CABG surgery. JAMA 2012; 308: 512-3.

27. Nasso G, Popoff G, Lamarra M, Romano V, Coppola $\mathrm{R}$, Bartolomucci F, et al. Impact of arterial revascularization in patients undergoing coronary bypass. J Card Surg 2012; 27: 427-33.

28. Buxton BF, Shi WY, Tatoulis J, Fuller JA, Rosalion A, Hayward PA. Total arterial revascularization with internal thoracic and radial artery grafts in triple-vessel coronary artery disease is associated with improved survival. J Thorac Cardiovasc Surg 2014; 148 (4): 1238-43.

29. Locker C, Schaff HV, Daly RC, Dearani JA, Bell MR, Frye RL. Multiple arterial grafts improve survival with coronary artery bypass graft surgery versus conventional coronary artery bypass grafting compared with percutaneous coronary interventions. J Thorac Cardiovasc Surg 2016; 152 (2): 369-79.

30. The Society of Thoracic Surgeons. Adult Cardiac Surgery Database. Chicago: The Society; 2014.

31. Huffmyer J, Raphael J. The current status of off-pump coronary bypass surgery. Curr Opin Anaesthesiol 2011; 24: 64-9.

32. González R, Seguel E, Stockins A, Campos R, Neira L, Alarcon E. Cirugía Coronaria: Revascularización miocárdica sin circulación extracorpórea. Rev Chil Cir 2009; 61: 578-81.

33. Seguel E, González R, Stockins A, Alarcón E, Concha R. Resultados inmediatos y a mediano plazo de la cirugía coronaria sin circulación extracorpórea. Rev Med Chile 2013; 141: 281-90.

34. Nashef SA, Roques F, Michel P, Gauducheau E, Lemeshow S, Salamon R. European system for cardiac operative risk evaluation (EuroSCORE). Eur J Cardiothorac Surg 1999; 16 (1): 9-139.

35. Nashef SA, Roques F, Hammill BG, Peterson ED, Michel P, Grover FL, et al. Validation of European System for Cardiac Operative Risk Evaluation (EuroSCORE) in North American cardiac surgery. Eur J Cardiothorac Surg 2002; 22 (1): 101-5.

36. Nashef SA, Roques F, Sharples LD, Nilsson J, Smith C, Goldstone AR, et al. EuroSCORE II. Eur J Cardiothorac Surg 2012; 41 (4): 734-44.

37. Alderman EL, Fisher LD, Litwin P, Kaiser GC, Myers WO, Maynard C. Results of coronary artery surgery in patients with poor left ventricular function (CASS). Circulation 1983; 68: 785-95.

38. Passamani E, Davis KB, Gillespie MJ, Killip T. A rando- 
mized trial of coronary artery bypass surgery. Survival of patients with a low ejection fraction. N Engl J Med 1985; 312: 1665-71.

39. Di Carli MF, Maddahi J, Rokhsar S, Schelbert HR, Bianco-Batlles D, Brunken RC. Long-term survival of patients with coronary artery disease and left ventricular dysfunction: implications for the role of myocardial viability assessment in management decisions. J Thorac Cardiovasc Surg 1998; 116: 997-1004.

40. Velazquez EJ, Lee KL, Deja MA, Jain A, Sopko G, Marchenko A, et al. Coronary artery bypass surgery in patients with left ventricular dysfunction. N Engl J Med 2011; 364: 1607-16.

41. Velázquez EJ, Lee KL, Jones RH, Al-Khalidi H, Hill JA, Panza JA, et al. Coronary-Artery Bypass Surgery in Patients with Ischemic Cardiomyopathy. N Engl J Med 2016; 374: 1511-20.

42. Ascione R, Narayan P, Rogers CA, Lim KH, Capoun R, Angelini GD. Early and midterm clinical outcome in patients with severe left ventricular dysfunction undergoing coronary artery surgery. Ann Thorac Surg 2003; 76: 793-9.

43. Trachiotis GD, Weintraub WS, Johnston TS, Jones EL, Guyton RA, Craver JM. Coronary artery bypass grafting in patients with advanced left ventricular dysfunction. Ann Thorac Surg 1998; 66: 1632-9.

44. Bouchart F, Tabley A, Litzler PY, Haas-Hubscher C, Bessou JP, Soyer R. Myocardial revascularization in patients with severe ischemic left ventricular dysfunction. Long term follow-up in 141 patients. Eur J Cardiothorac Surg 2001; 20: 1157-62.

45. Irarrázaval MJ, Muñoz C, Garayar B, Morán S, Zalaquett R, Maturana G, et al. Cirugía Coronaria. Veinte años de seguimiento. Rev Med Chile 1998; 126: 63-74.

46. Morán S, Irarrázaval MJ, Zalaquett R, Villavicencio M, Garayar B, Muñoz C, et al. Revascularización miocárdica con una o dos arterias mamarias: resultados clínicos y seguimiento alejado. Rev Med Chile 1997; 125: 391-401. 